\title{
The linearity of the volume/pressure response during intracranial pressure "reserve" testing
}

\author{
H A R OL D A W ILKINSON, STEVEN ROSENFELD, \\ DEBORAH DENHERDER, AND RODERICK BRONSON
}

From the Neurosurgery Department, University of Massachusetts Medical Center and the Department of Comparative Pathology, New England Regional Primate Research Center

SUMMAR Y The intracranial pressure "reserve" test seems to be the most reliable method of determining when the brain's natural mechanisms for pressure compensation for added intracranial volume have been compromised or exhausted. The test employs a timed sequence of intracranial fluid injections, but as a safety precaution injections are discontinued if intracranial pressure remains elevated more than 10 Torr over baseline. In this case, a linear extrapolation is then calculated to determine the elevation which might have been achieved by a full series of injections. However, this linear extrapolation has been criticised on the expectation that an exponential response should be expected. A series of experimental observations in dogs and baboons and a review of clinical records in humans have been made to determine the observed slope of increase following aliquot injection during performance of the intracranial pressure reserve test. In these species the observed response was actually linear in shape rather than exponential. This held true even for different initial baseline values and with different volumes of "lesion" balloon inflations in experimental animals. A theoretic explanation is proposed.

The intracranial pressure (ICP) reserve test has been put forward as a laboratory and clinical test for measuring the brain's ability to maintain intracranial pressure constant following changes in intracranial volume. ${ }^{1-3}$ At one minute intervals a given aliquot of fluid is administered subdurally or intraventricularly. The pressure elevation achieved at the end of five minutes is taken as a measure of the brain's reserve capacity for volume/pressure adaptation. A special modification of the test is recommended for safe use in patients; sequential injections are stopped 60 seconds after the last injection if the pressure elevation remains greater than 10 Torr. The rise in pressure which might have been achieved had the sequence been continued to a full five injections is linearly extrapolated as a "slope" value. The choice of a linear extrapolation has been challenged as inaccurate since most previous

Address for reprint requests: Professor HA Wilkinson, Department of Neurosurgery, University of Massachusetts Medical Center, 55 Lake Avenue North, Worcester MA 01605, USA.

Accepted 15 May 1980 studies on the volume/pressure response predict a complex exponential rise with further injection..$^{-9}$ The present study was undertaken to test the validity of this safety modification of the ICP reserve test.

\section{Methods}

Experiments were undertaken in dogs and baboons and clinical tracings from human patients were analysed retrospectively.

Studies in dogs A series of nine mongrel dogs with brain volumes of approximately $150 \mathrm{ml}$ were operated upon under pentobarbital general anaesthesia. Arterial blood pressure was monitored continuously on a polygraph via femoral artery catheters. Urine volume and specific gravity, serum electrolytes and arterial blood gases were monitored at intervals. All animals were intubated but artificial respiration was added only if significant blood gas disturbances developed. Intracranial pressure was recorded continuously from the left hemisphere both by an 
intraventricular catheter and by subdural ICP monitoring cup catheter. An $8 \mathrm{ml}$ volume "lesion" balloon was placed epidurally over the right hemisphere and a $3 \mathrm{ml}$ ICP reserve test balloon was placed subdurally over the left hemisphere. ICP reserve testing was performed by rapid injection into the reserve test balloon of $0.1 \mathrm{ml}$ aliquots of fluid at 60 second intervals for a total of five injections (See fig 1). The ICP reserve test was repeated at 20 minute intervals. The lesion balloon was inflated with 0.5 or $1.0 \mathrm{ml}$ aliquots of saline injected at 20 or 40 minute intervals until a sustained ICP greater than 40 Torr was achieved.

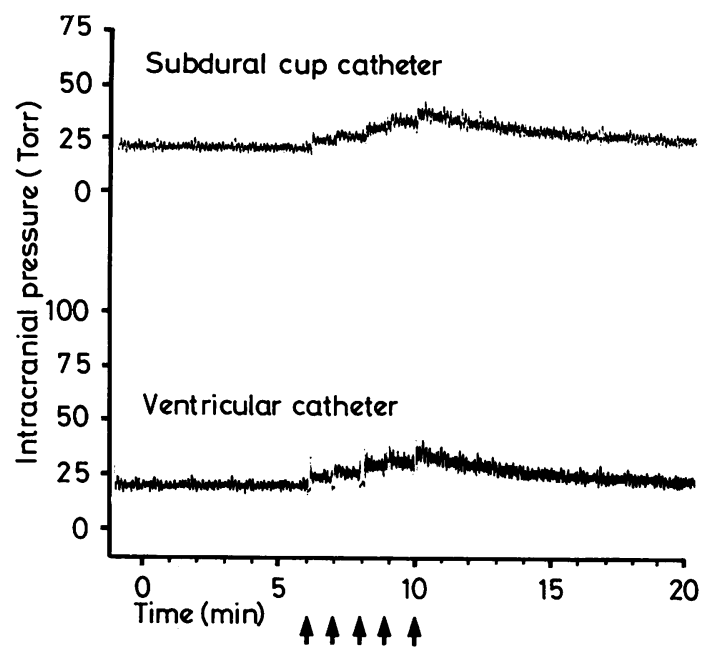

Fig 1 ICP reserve test in a dog, using five intraventricular injections of $0.1 \mathrm{ml}$ Ringer's lactate solution at 60 second intervals (arrows). Resulting increase in ICP was measured with both a ventricular catheter and a subdural cup catheter. The pressure increase achieved 60 seconds after the last injection was 15.0 Torr, a quantitative measurement of the brain's "reactivity" to volumetic stress.

Studies in baboons The format of experimental surgery and monitoring for the baboons was almost identical to that employed for the dogs, though these experiments were carried out at the New England Regional Primate Research Center. Five adult baboons with brain volumes of approximately $250 \mathrm{ml}$ were employed. For the ICP reserve test aliquots of $0.25 \mathrm{ml}$ were injected. The lesion balloon was inflated with 1.0 or $2.0 \mathrm{ml}$ aliquots of saline at 20 minute intervals until a sustained ICP greater than 30 Torr was achieved.

Studies in humans Tracings of continuous ICP monitoring were analysed from our most recent series of 11 patients undergoing postoperative ICP monitoring immediately following craniotomy for a variety of clinical diagnoses: trauma-4, tumour-4, aneurysm-2, intracerebral haemorrhage-1. In each of these patients clinical ICP reserve testing was performed once or twice a day, using aliquot injections of $1.0 \mathrm{ml}$ of Ringer's lactate solution with bacitracin delivered into the subdural space through ICP monitoring cup catheters, as described in our earlier publications. ${ }^{12}$ As a safety precaution ICP reserve testing was not done if baseline ICP exceeded 30 Torr, and a full series of five injections was given only if the resultant rise in ICP did not exceed 10 Torr. For the purpose of this study only those ICP reserve tests in which a full series of five injections had been given were analysed. Baseline ICP readings in these patients averaged only 9.8 Torr, though baseline ICP values between 20 and 30 Torr were encountered in 12 reserve tests done in five patients.

Data analysis Data obtained from dogs and baboons was analysed as grouped data according to initial ICP level or according to lesion balloon volume. For the dogs the following initial ICP ranges were used: $0-10$ Torr, 10.5 to 20 Torr, 20.5 to 30 Torr and 30.5 Torr or greater. For the baboons initial pressure ranges were: 0 to 1 Torr, 10.5 to 20 Torr and greater than 20 Torr. (Since these animals were to be used for subsequent additional studies higher pressure ranges were not generated for fear of causing death.) For the dogs, lesion balloon volume data was grouped as: $0 \mathrm{ml}, 0.5$ to $1.5 \mathrm{ml}$, and $2 \mathrm{ml}$ or greater. For the baboons, lesion balloon volume ranges were: $0 \mathrm{ml}, 1$ to $3 \mathrm{ml}$, and $4 \mathrm{ml}$ or more. Data from humans was grouped as those reserve tests with initial ICP's below 15 Torr and those with initial ICP's between 15 and 30 Torr. Because of the need for safety in human therapy only patients with baseline ICP's below 30 Torr could be included within the design of this study.

Means and standard deviations and standard errors of the means were calculated for each grouped set of data.

\section{Results}

In general, there was good concordance between results observed from the intraventricular catheter and from the subdural monitoring catheter, so data from both devices was pooled. At higher pressure ranges the ventricular catheter stopped working at times, so ventricular catheter data from ICP reserve testing during these 


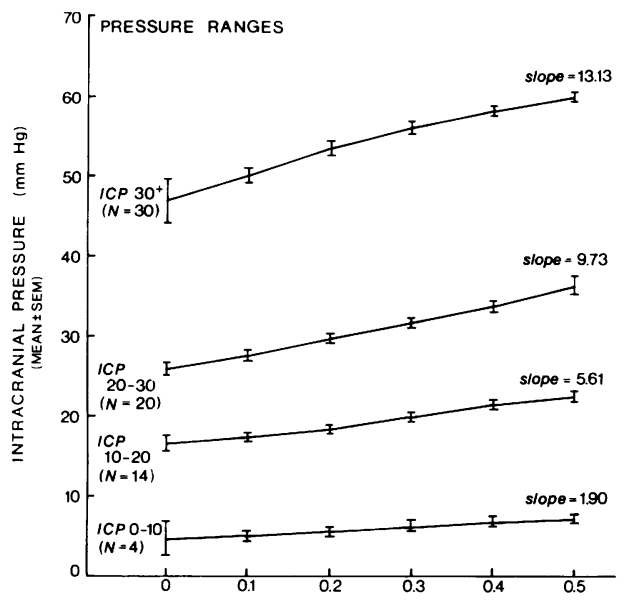

VOLUME INJECTED (ml) FOR RESERVE TEST

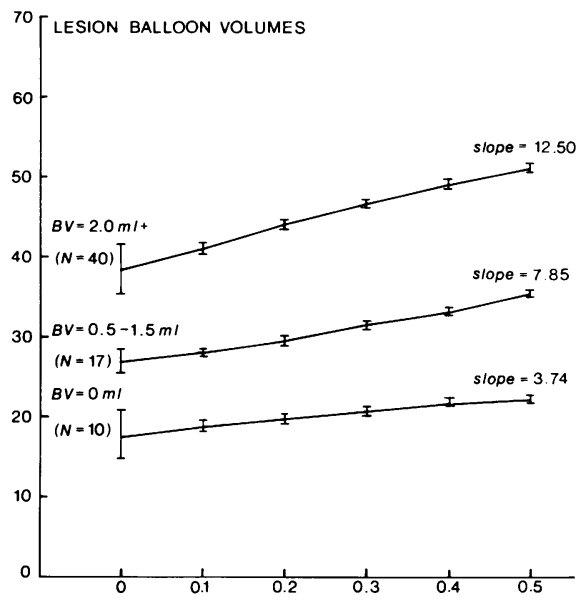

VOLUME INJECTED (ml) FOR RESERVE TEST

Fig 2 Mean ICP responses to sequential intracranial injections of $0.1 \mathrm{ml}$ volumes in dogs, with data grouped according to initial ICP (left) or lesion balloon volume (" $\mathrm{BV}$ ", right). Bars denote means \pm standard errors of means.

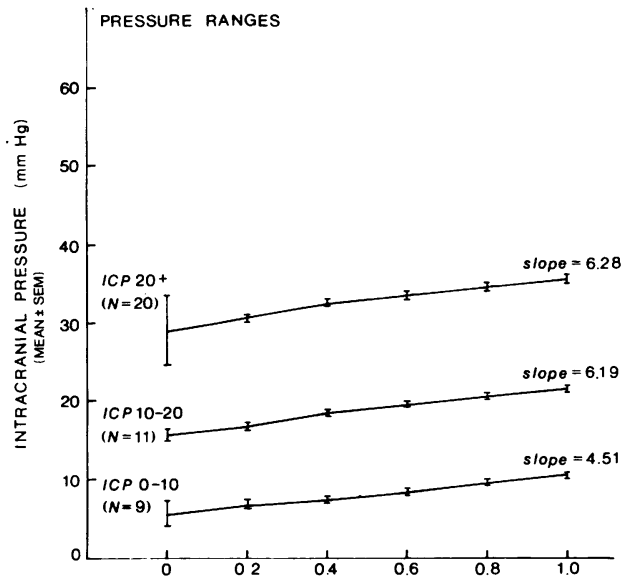

VOLUME INJECTED (mI) FOR RESERVE TEST

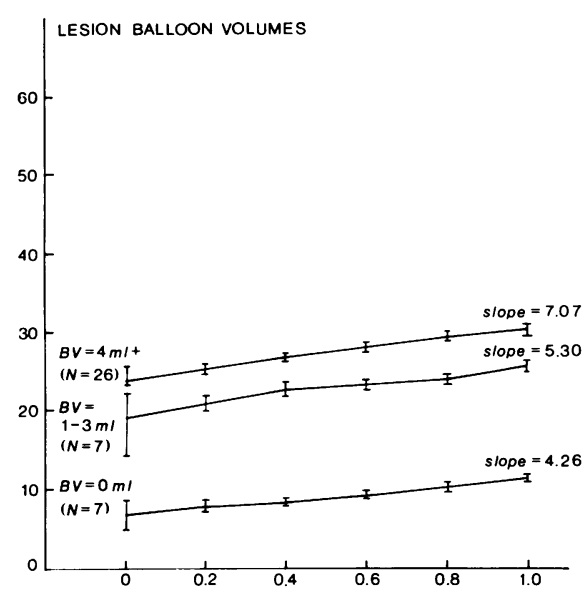

VOLUME INJECTED (mI) FOR RESERVE TEST

Fig 3 Mean ICP responses to sequential intracranial injections of $0.25 \mathrm{ml}$ volumes in baboons, with da:a grouped according to initial ICP (left) or lesion balloon volume (" $B V$ ", right).

episodes is not included. A total of 67 reserve tests were analysed in dogs, 40 reserve tests in baboons and 42 tests in patients.

As can be seen from figs 2 to 4 , ICP volume/ pressure responses following sequential injections at one minute intervals rose in a remarkably linear fashion in each species and in each of the different sub-groups of pooled data. Figure 5 con- firms this linearity. In all three species studied, the volume/pressure response to each aliquot injected became progressively greater with increasing initial ICP, and became progressively greater with increasing lesion balloon volume in the two experimental animal studies (figs 2 and 3 ). At higher levels of initial ICP, and with larger lesion balloon volumes, the volume/pressure response 
actually rose in a slightly inversely exponential fashion rather than demonstrating a positive exponential curve.

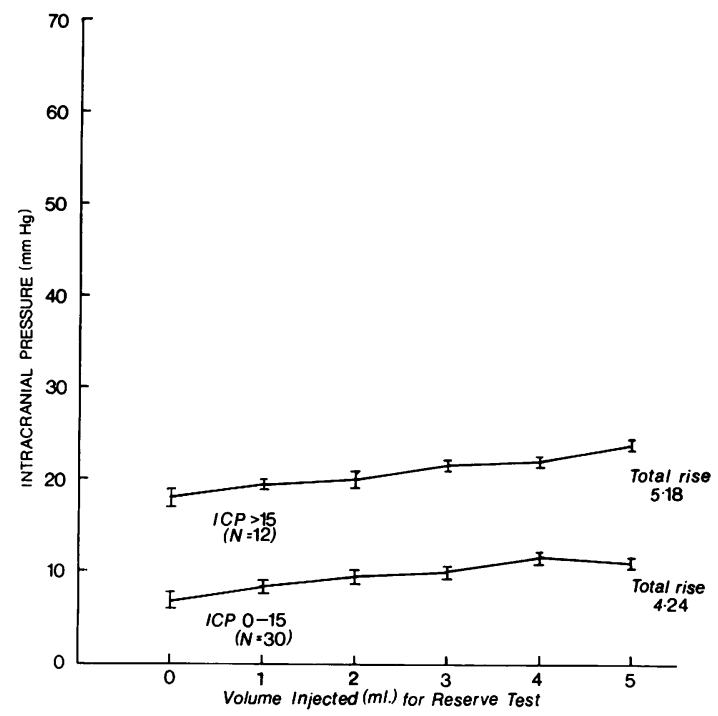

Fig 4 Mean ICP responses to sequential intracranial injections of $1.0 \mathrm{ml}$ volumes in humans, with data grouped according to initial ICP.

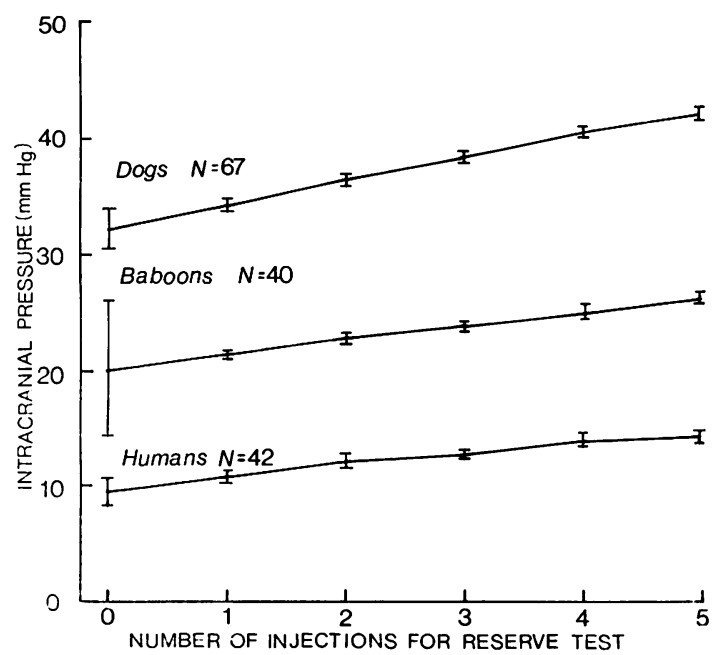

Fig 5 Mean ICP responses to sequential intracranial injections, displaying pooled data for three mammalian species (including all determinations, irrespective of initial ICP or lesion balloon volume). Volumes injected same as in figs 2 to 4.

\section{Discussion}

The Monroe-Kellie doctrine, now nearly 200 years old, ${ }^{10}$ describes the central nervous system as a "closed box" in which any increase in volume will result in a rise in intracranial pressure (ICP). More recently it has been recognised that the brain possesses a series of adaptive mechanisms for controlling the rise in ICP which results from the volume increases. ${ }^{11}$ Current understanding of the complexities of the intracranial volume/pressure response stems largely from the studies of Langfitt, ${ }^{4}$ :9 Löfgren, ${ }^{7}$ Marmarou ${ }^{1213}$ and Miller. ${ }^{811}$ All reported an exponential rise in ICP as a result of progressive increase in intracranial fluid volume in studies with relatively rapid or continuous fluid injections. Langfitt ${ }^{4}$ noted that the shape of the curve would be altered by the rate of injection, but a generally exponential shape was still described. Miller ${ }^{8}$ 14-16 $^{-1}$ pointed out the variations in the slope of this curve in pathological conditions. $\mathrm{He}$ also suggested a clinical adaptation of volume/pressure testing as a bedside procedure to estimate the degree of impairment of the brain's normal adaptive mechanisms. Miller's test of "compliance" or "elastance" is performed by stressing the brain volumetrically with a $1 \mathrm{ml}$ intracranial injection of fluid (in humans) and measuring the immediate change in ICP which results.

In attempting to apply Miller's suggestion we found his rapid test of "compliance" or "elastance" to be too inaccurate and too difficult to measure for routine work. Subsequently we undertook a study in dogs which led to the description of the "ICP reserve test", ${ }^{1}$ which is an expansion of Miller's test. The brain is stressed volumetrically over a five minute time span to test both the brain's rapidly acting pressure corrective capabilities, which are measured by tests of "elastance," and its less rapidly acting capabilities. As applied clinically, this test consists of rapid subdural or intraventricular injections of $1.0 \mathrm{ml}$ aliquots of fluid at 60 second intervals. Subdural injections of fluid carry the risk of actifactually low results if fluid leakage out of the subdural space occurs, but obvious fluid leakage has been encountered only rarely in patients in whom we employ ICP monitoring cup catheters and occasional subdural "Richmond bolts." The increase in ICP achieved 60 seconds following the final injection is taken as a measurement of the brain's "reactivity" to this form of volumeiric stress testing." This measurement of "reactivity" is independent in experimental animals of initial 
ICP and reflects the volume of an experimental mass lesion more closely than initial ICP alone. ${ }^{13}$

Injections of additional volume inside the head of patients with central nervous systems lesions carries the potential risk of increasing ICP to produce secondary brain herniation. So the ICP reserve test for clinical use includes a safety modification. During performance of the test the resultant ICP increase is noted 60 seconds following each of the aliquot injections, and if ICP remains elevated more than 10 Torr over the initial pressure no further injections are given. Instead the rise in pressure which might have been achieved following the full series of five injections is calculated as a simple linear slope. Thus an observed increase of 12 Torr following three injections would be calculated as a "slope value" of 20 Torr $(12 / 3 \times 5=20)$.

The accuracy and reliability of this simple linear computation have been challenged because previous studies have reported exponential rather than linear pressure rises with rapid or continuous fluid infusions. ${ }^{4-8} 13$ When the ICP reserve test was initially published it was felt that the resultant "slope value" would represent a useful, albeit probably somewhat conservative, quantitative estimate of the degree of exhaustion of the brain's reserve capabilities. We have shown now that in at least three species of mammals (dog, baboon and human) the ICP response obtained from a timed series of aliquot injections is in fact a linear rise rather than an exponential one.

We have not been able to analyse the reasons why this observed result occurs, but we suggest the following hypothesis. It is easy to demonstrate that ICP falls in an inversely exponential fashion over five to 15 minutes following sudden inflation of an intracranial balloon in the closed cranial cavity of an experimental animal. With the addition of a second intracranial mass lesion (balloon inflation or ICP reserve test aliquot injection) 60 seconds following the first volumetric stress an additive result should be observed, with compensation for the second volumetric event being superimposed upon that already underway following the first event. As a result, by the fifth injection a series of additive compensations should have taken place which may well cancel out the predicted exponentially increasing shape of the volume pressure curve as observed with continuous or rapid infusions (fig 6).

Conclusion The timed sequential nature of the volumetric stress testing used in the ICP reserve test results in a linear increase in ICP in at least three mammalian species, rather than the expo-
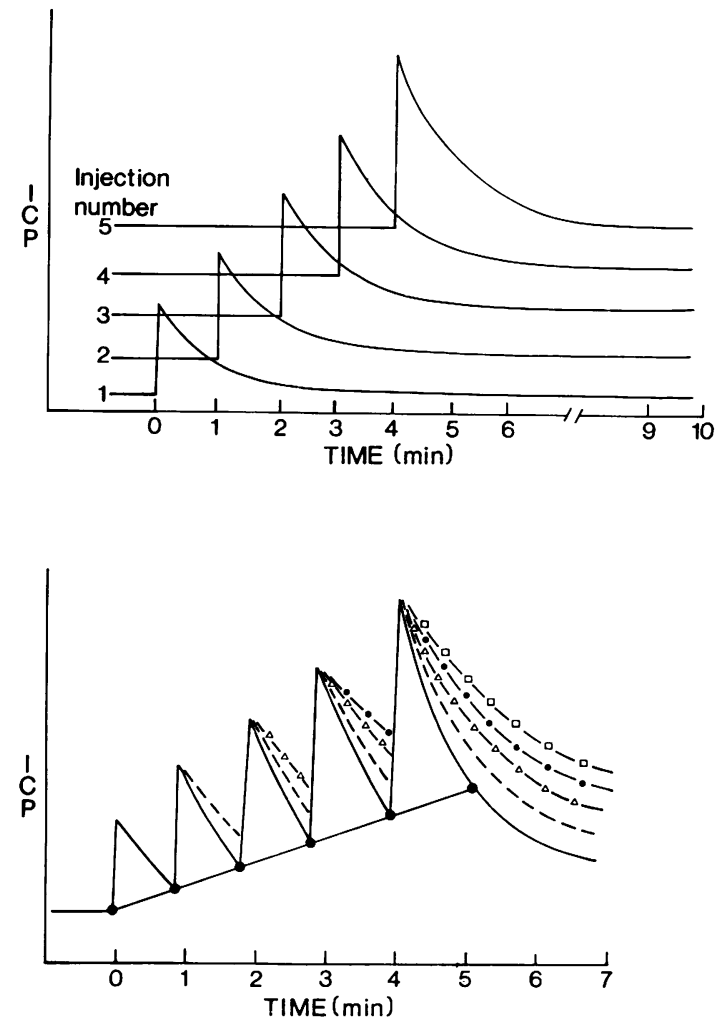

Fig 6 Diagram of theoretical explanation for linearity of ICP response to timed sequential intracranial fluid injections (Top): Each injection would be expected to cause an abrupt rise, then an inverse exponential fall in ICP over a five to 15 minute period. With higher baseline ICP's a greater rise in ICP would be expected. (Bottom): With repeated injections at one minute intervals the reductions in ICP triggered by each injection would be expected to have a cumulative effect. (Heavy solid line denotes observed ICP; broken lines represent cumulative reductions following prior injections).

nential rise expected from earlier studies. As a result, the "slope value" calculated during the clinical performance of the ICP reserve test should be an even more accurate determination of ICP "reserve" than initially expected.

\section{References}

1 Wilkinson HA, Arredondo D, Weems S, Kaner D. Intracranial pressure reserve testing; a study in experimental animals. Arch Neurol 1978; 35: 567-76. 
2 Wilkinson HA. Intracranial pressure reserve testing: initial clinical observations. Arch Neurol 1978; 35:661-7.

3 Wilkinson HA, Schuman N, Ruggiero J. Nonvolumetric methods of detecting impaired intracranial compliance or reactivity: pulse width and wave form analysis. $J$ Neurosurg $1979 ;$ 50:758-67.

4 Langfitt TW. Pathophysiology of increased ICP. In: Brock M, Dietz H, eds. Intracranial Pressure: Experimental and Clinical Aspects. New York: Springer-Verlag, 1972: 361-4.

5 Langfitt TW, Weinstein JD, Kassell NF. Cerebral vasomotor paralysis produced by intracranial hypertension. Neurology (Minneap) 1965; 15: 622-41.

6 Leech PJ, Miller JD. Intracranial volume/pressure relationships during experimental brain compression in primates. I. Pressure response to changes in ventricular volume. $J$ Neurol, Neurosurg Psychiatry 1974; 37:1093-8.

7 Löfgren J, Von Essen C, Zwetnow NN. The pressure-volume curve of the cerebral fluid space in dogs. Acta Neurol Scand 1973; 49:557-74.

8 Miller JD, Leech PJ. Assessing the effects of mannitol and steroid therapy on intracranial volume-pressure relationships in patients. $J$ Neurosurg 1975; 42:274-81.

9 Weinstein JD, Langfitt TW, Kassell NF. Vasopressor response to increased intracranial pressure. Neurology 1964; 114:1118-31.
10 Monroe A. In: Creech and Johnson, eds. Observations on the structure and function of the nervous system. Edinburgh: 1783.

11 Miller JD, Sullivan HG. Severe intracranial hypertension. In Trubuhovich RV, ed. Management of acute intracranial disasters. Boston: Little, Brown \& Co 1979: 19-75.

12 Marmarou A, Shulman K. Computer modeling of CSF pressure/volume and its relationship to hydrocephalus. In: Brock $M$, Dietz $H$, eds. Intracranial Pressure: Experimental and Clinical Aspects. New York: Springer-Verlag 1972: 275-9.

13 Marmarou A, Shulman K, LaMorgese J. Compartmental analysis of compliance and outflow resistance of the cerebrospinal fluid system. $J$ Neurosurg 1975; 43:523-34.

14 Miller JD, Garibi J, Pickard JD. Induced changes of cerebrospinal fluid volume: Effects during continuous monitoring of ventricular fluid pressure. Arch Neurol 1973; 28:265-9.

15 Leech PJ, Miller JD. Intracranial volume/ pressure relationships during experimental brain compression in primates. II. Effects of induced changes in arterial pressure. J Neurol, Neurosurg Psychiatry 1974; 37:1099-104.

16 Leech PJ, Miller JD. Intracranial volume/ pressure relationships during experimental brain compression in primates. III. The effect of mannitol and hypocapnia. J Neurol, Neurosurg Psychiatry 1974; 37:1105-11. 